\title{
Patient Selection
}

\author{
Klaus Ueberreiter
}

The general precondition for transplantation of large volumes of tissue is that suction-friendly deposits must exist, and these are generally present starting from a body mass index or BMI of above 18-20. However, when more fat tissue is available for use, the execution of this job gets simplified when more fat tissue is available for use.

\section{- In the ideal case, the patient should have a BMI of between 22 and 30 .}

Very favorable preconditions would exist, if, on the one hand, disturbing fat deposits are present on the upper thighs, at the stomach or on the hips, and very small breasts. In these cases, an aesthetic improvement is combined by reduction of unwelcom deposits with an aesthetic improvement of the breasts.
No proof has been produced so far that fat from a particular region of the body is suitable for transplantation. In practice, we recommend to follow the preference indicated by the patients themselves.

But from experience, there is a basis for assumption that the tissue retains the properties of its site of origin, that is to say, especially stubborn fat from the area of the abdomen retains its tendency to increase in the breast area in case of weight gain.

- Lipedema fat general has a lower take rate and is not recommended for grafting.

- Many clinical users are of the unanimous opinion that smoking reduces the rate of healing, even though extensive studies have not been done to support this viewpoint.

\footnotetext{
K. Ueberreiter $(\bowtie)$

Park-Klinik Birkenwerder, Fachklinik für Plastische und Ästhetische Chirurgie, Birkenwerder, Germany

e-mail: dr@ueberreiter.com
} 